Torreón Universitario

www.faremcarazo.unan.edu.ni / ISSN 2410-5708 / e-ISSN 2313-7215

Año 9 | Núm. 26 | pág. 124 - pág. 146 | octubre 2020 - enero 2021
Esta obra está bajo una licencia de Creative Commons

Atribución-NoComercial-SinDerivar 4.0 Internacional

\title{
Indice de Posición Topográfica (TPI) para identificar áreas inundables y zonificación de especies vegetales en un manglar del Pacifico Sur de Nicaragua.
}

\author{
Dra. Heyddy Calderón \\ Ph.D en Hidrología \\ UNAN-Managua, Instituto de Geología y Geofísica \\ https://orcid.org/0000-0002-4405-8971 \\ heyddy.calderon@igg.unan.edu.ni \\ heyddy.calderon@gmail.com \\ MSc. José Enrique Pérez \\ Máster en Gestión Ambiental \\ UNAN-Managua \\ https://orcid.org/0000-0001-8456-3684
}

Dr. Marcel Chow

Ph.D en Ciencias Astrofísica

UNAN-Managua, Instituto de Geología y Geofísica

https://orcid.org/0000-0001-6073-9956

Lic. Juan Bautista Reyna

Ingeniería Geológica

UNAN-Managua

https://orcid.org/0000-0001-5065-8765

Fecha de recepción: 06 de febrero, 2020 / Fecha de aceptación: 07 de julio, 2020

https://doi.org/10.5377/torreon.v9i26.10264

Palabras clave: TPI, bosque de mangle, geomorfología,

zonas inundables, gestión integral.

\section{RESUMEN}

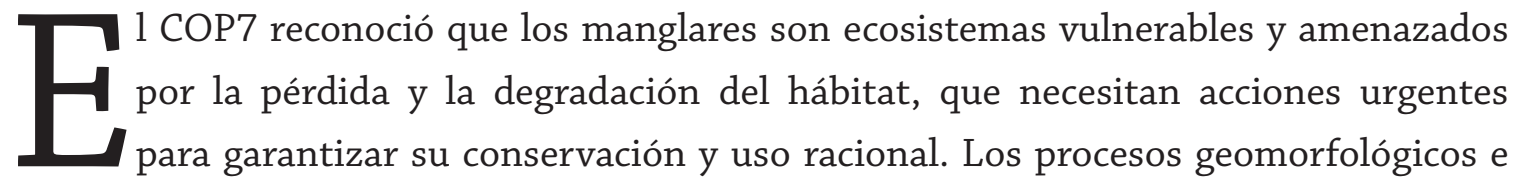
hidrológicos son claves para el crecimiento y desarrollo de los bosques de mangle. Sin embargo, muy pocos estudios al respecto existen en Centroamérica. Los bosques de manglar ofrecen valiosos servicios económicos y ecológicos, como turismo y albergue de especies de flora y 
fauna. Además, protegen ante el incremento del nivel del mar causado por el cambio climático. La hidrología del manglar del Río Ostional $\left(\sim 0.2 \mathrm{~km}^{2}\right)$, en el Pacífico Sur de Nicaragua, ha sido estudiado en años recientes sugiriendo una relación entre las especies de mangle y los aportes de agua dulce. En este proyecto, se exploró el uso del Índice de Posición Topográfica (TPI) para identificar zonas inundables a pequeña escala y con poca variación de elevación ( $3 \mathrm{~m})$ en un manglar. Para ello se realizó una caracterización geomorfológica mediante el levantamiento topográfico a detalle de la zona y análisis del TPI como método de clasificación de formas del terreno para identificar las zonas de inundación; así como el levantamiento de zonificación de especies vegetales. La clasificación del TPI fue relacionada a la zonificación de especies. Esta relación permitirá identificar las zonas más sensibles a modificaciones geomorfológicas de posible origen antrópico, lo cual es un insumo esencial para la gestión integral del bosque.

\section{INTRODUCCIÓN}

Centroamérica cuenta con unos $4000 \mathrm{~km}^{2}$ de mangles en la costa Pacífica, de los cuales Nicaragua posee $400 \mathrm{~km}^{2}$ (Ellison, 2004). El bosque de mangle de Ostional, ubicado en el municipio de San Juan del Sur, es un típico ejemplo de los ecosistemas costeros en el Pacífico Sur de Nicaragua. La presencia de manglares y barras de arena en la playa dominan los estuarios de estos ríos (Jiménez, 1992). La playa de Ostional se encuentra además muy cerca del Refugio de Vida Silvestre La Flor, y aunque en menor medida, también es sitio de anidación de tortugas marinas. Adicionalmente, el bosque de mangle refugia a otras especies faunísticas (Aves, monos, mamíferos pequeños) y es una atracción turística que genera ingresos económicos a la población de Ostional (Calderón, 2014).

El Examen Mundial de los Recursos de Humedales y las Prioridades para el Inventario de Humedales (COP7) reconoció que los manglares y los arrecifes de coral son algunos de los ecosistemas más vulnerables y amenazados por la pérdida y la degradación de los hábitat, y por consiguiente necesitan acciones urgentes y prioritarias para garantizar su conservación y uso racional. Por otra parte, los procesos geomorfológicos e hidrológicos son claves para el crecimiento y desarrollo de los bosques de mangle (Feller y Sitnik, 1996; He et al., 2010; Jiménez, 1994,1999; Lugo y Snedaker, 1974, Tomlinson, 1986). Este sistema ha sido empezado a estudiar desde el punto de vista hidrológico y durante ese proceso se han identificado de manera general patrones de zonificación de especies de mangle (Calderón, 2015; Calderón et al 2014, De Vries 2016). Sin embargo, es necesario profundizar más en el entendimiento entre los flujos de agua superficial hacia el sistema y su relación con la zonificación de especies de mangle. Este conocimiento es necesario para identificar las áreas del mangle más sensibles a cambios morfológicos de origen natural o antrópico, como alteración al relieve natural, aparición de nuevas cárcavas, descarga de aguas grises domésticas o desechos sólidos dentro del manglar. 
Por otro lado, no existe un mapa de zonificación de especies, lo cual es esencial para poder generar un plan de gestión integral del manglar. Además, las zonas de inundación son particularmente importantes dado que el crecimiento y desarrollo del bosque dependen en gran medida del delicado balance entre los flujos de agua dulce y los flujos de agua salada, lo que también determina la zonificación de especies. Este trabajo pretende contribuir al entendimiento de los procesos hidrológicos en los sistemas de mangle de Centroamérica y generar insumos necesarios para el Plan de Gestión Integral del bosque de Mangle de Ostional. El objetivo de este proyecto es probar el uso del TPI para clasificar formas de terreno inundables a pequeña escala y con poca variación de elevación ( $3 \mathrm{~m})$ en un manglar del suroeste de Nicaragua, para correlacionarlas con patrones de zonificación de especies de mangle.

\section{Área de Estudio}

Ostional es una pequeña comunidad rural costera del municipio de San Juan del Sur, Rivas. Está ubicado en la zona de vida de Holdrige de bosque tropical seco. La zona de estudio es una planicie de inundación de alrededor de $0.5 \mathrm{~km}$ de ancho caracterizada por un estero ciego, cerrado por una barra de arena producida estacionalmente por la marea de "spring". Durante el pico de la época lluviosa (Octubre-Noviembre) la crecida del río Ostional rompe la barra liberando el agua contenida en el estuario (deVries, 2016). La presencia de la barra de arena ayuda a mantener el balance hídrico positivo durante la época de estiaje o de sequías, lo cual favorece la subsistencia y crecimiento de las especies de mangle (Calderón et al., 2014). El suelo es arcilloso y el nivel freático oscila entre 1 y $0.5 \mathrm{~m}$ durante el año. Las especies de mangle identificadas en la zona de estudio son blanco, rojo, piñuela y botoncillo (deVries 2016).

La población local es de alrededor de 1500 personas y la economía está basada en la pesca, turismo y agricultura de subsistencia (Calderón, 2014). La zona del manglar es uno de los atractivos turísticos ofrecidos a los visitantes para el avistamiento de aves, mamíferos, lagartos, senderismo y kayaking. Sin embargo, también es utilizada como fuente de leña y pastizaje.

\section{METODOLOGÍA}

\section{Caracterización y zonificación de especies vegetales}

El levantamiento se realizó en el estuario Ostional haciendo puntos aleatorios tratando de dar un porte homogenizado del lugar. El estudio es de carácter descriptivo y de corte transversal, elaborado del 28 al 30 de octubre del 2015, tiempo suficiente que permitió determinar la presencia y diversidad de las especies vegetales existentes en los puntos muestreados, dada la característica de muestreo rápido de esta investigación. La población de estudio fueron todas las especies vegetales del reducto de bosque, vegetación asociada con el manglar presente en transectos detallados ajustándonos a los biotipos de árboles, arbustos y hierbas. 
Se realizó un muestreo de seis parcelas de 10 x $10 \mathrm{~m}\left(100 \mathrm{~m}^{2}\right)$, dos por cada formación vegetal, con la intención de obtener áreas homogéneas. Este método fue propuesto por Foster et al., (1995), para realizar evaluaciones rápidas de la vegetación. Se consideró que usualmente es mejor hacer varios muestreos pequeños que pocos grandes. Foster et al., (1995) mencionan que 50 individuos para cada muestra puede ser un número adecuado, con el cual se pueden hacer varios muestreos representativos en un solo día (Mateucc y Colma, 1982; Mostacedo \& Fredericksen, 2000; Araujo et al., 2005). La muestra, fue analizada con índices de biodiversidad para caracterizar el manglar y su flora asociada, se emplearon los índices de Margalef para conocer la riqueza, índice de Shannon-Weinner para medir la diversidad e índice de Simpson, para la evaluar la dominancia de las especies. Las especies fueron identificadas con la bibliografía Flora de Nicaragua (Steven et al., 2001).

Índices de Biodiversidad

$$
\begin{gathered}
\left(\mathrm{H}^{\prime}\right)=-\sum_{i=1}^{1} P i * \operatorname{Ln} \mathrm{Pj} \\
\mathrm{D}=1 / \sum_{i=1}^{s} P i^{2} \\
\mathrm{DMg}=\frac{s-1}{\ln s}
\end{gathered}
$$

Donde:

$\mathbf{H}^{\prime}$ : Índice de diversidad Shannon -Wiener

D: Índice de dominancia de Simpson

$\mathbf{D M g}=$ Índice de riqueza de Margalef

Pi: ni/S

Ni: Número de individuos

Pi: Abundancia relativa de la especie i (pi: ni/N)

$\mathbf{N}$ : número total de individuos

S: riqueza especifica

इni: N

\section{Levantamiento topográfico a detalle}

El levantamiento topográfico a detalle se hizo con una estación total Leica Ts09 plus. Se utilizaron como benchmarks las elevaciones de tres piezómetros ubicados en la costa en el sitio 
de estudio. Esas elevaciones fueron levantadas en 2012 con un GPS diferencial marca Ashtech Locus L1 con precisión horizontal de $5 \mathrm{~mm}$ y vertical de $10 \mathrm{~mm}$. Para el levantamiento de los datos se orientó la estación total con el método de coordenadas conocidas, debido a la estructura de los manglares se utilizó un prisma con bastón de $3 \mathrm{~m}$ de alcance para lecturas altas y mini prisma de $0.6 \mathrm{~m}$ para lecturas bajas, las lecturas altiplanimétricas se realizaron en combinación con mediciones láser prisma y láser rebote. Se levantaron un total de 433 puntos tratando de distribuirlos uniformemente en el área. Las elevaciones oscilan entre 0.175 msnm a 3.104 msnm. Sin embargo en algunos sitios la densidad local de árboles dificultaba el levantamiento por lo que esas zonas tienen una menor densidad de puntos (Figura 1). La cantidad de puntos levantados topográficamente está condicionada por la densidad de árboles, lo que impide la visualización del prisma. En algunos sitios con menos individuos fue posible tener una mayor densidad de puntos.

\section{Generación del Modelo Digital de Terreno (DEM)}

El modelo digital de elevaciones fue generado desde los datos recolectados en el levantamiento topográfico a detalle (Figura 1). Estos datos fueron modelados usando el software estadístico R. Para la elección del tamaño del pixel, se realizó un análisis estadístico de la desviación estandar de cada punto con sus vecinos más cercanos (cinco vecinos para asegurar robustez estadística). De acuerdo a la distribución de las desviaciones estándar de estos datos se obtuvo un máximo en $2.5 \mathrm{~m}$, por lo que se estableció este valor como el tamaño de la celda para la generación del DEM.

El DEM fue generado usando una interpolación del tipo kriging simple, empleando la biblioteca geoestadística GSTAT de R y los datos recogidos del levantamiento topográfico. El método de interpolación Kriging es muy preciso y eficiente con respecto a otros métodos de interpolación en la creación de modelos digitales de elevación, proporciona análisis más robustos y con fundamentos estadísticos (Villatoro, 2008; Vílchez, 2000; Pineda M, 2015), por lo que este método fue el utilizado en este estudio. Como se mencionó anteriormente, fue elegido un tamaño de celda de 2.5 x $2.5 \mathrm{~m}$. El variograma fue ajustado usando un modelo esférico. La selección de otros modelos no produjo cambios significativos en el DEM. 

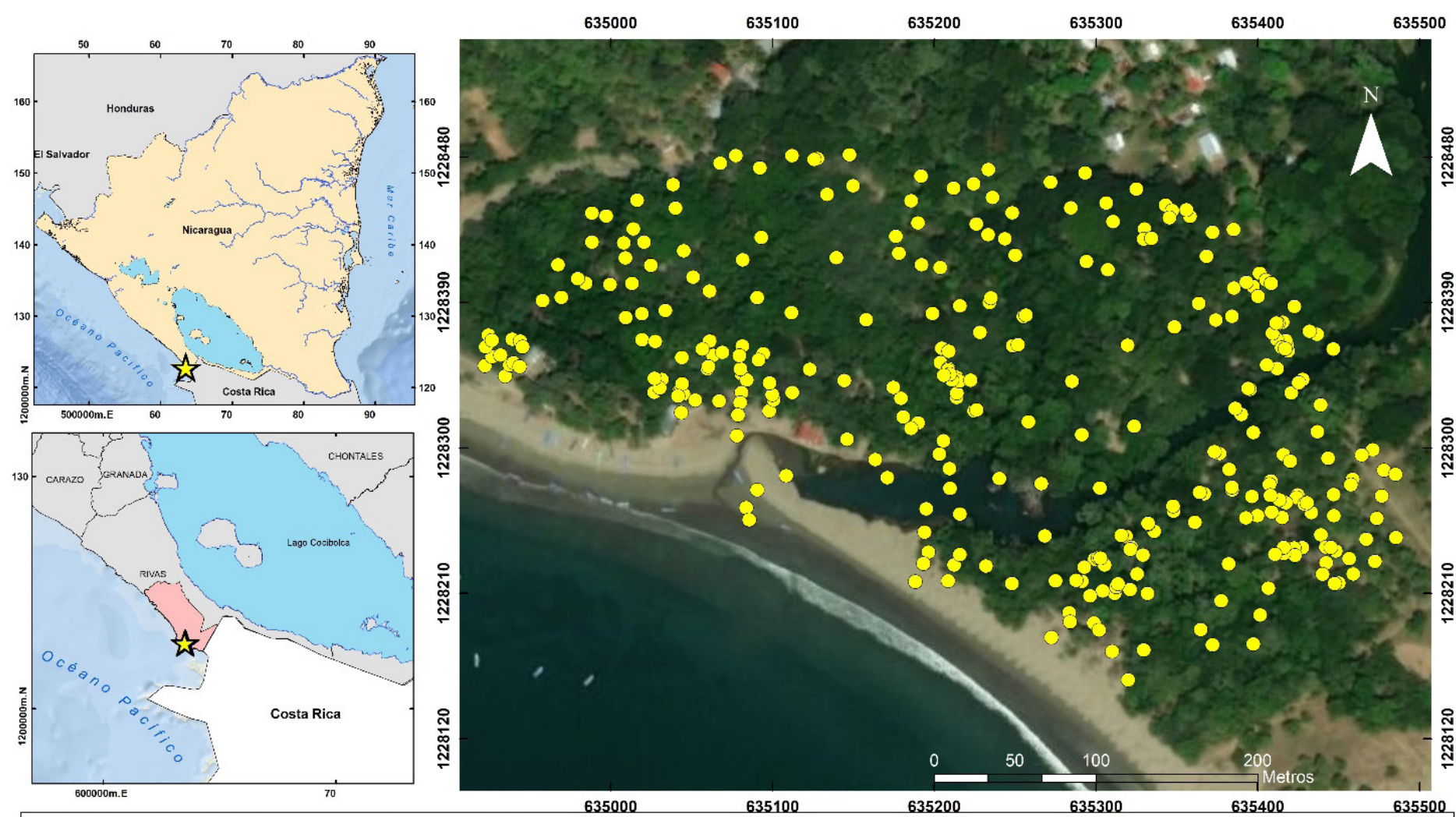

Sistema de Coordenadas: WGS 1984 UTM Zona N Proyección: Transversal de Mercator

Datum: WGS 1984

Este Falso: $500,000.000$

Norte Falso: 0.0000

Meridiano Central: -87.0000

Scale Factor: 0.9996

$1 \mathrm{~cm}$ equivale a 20 metros

eyenda

Latitud de Origen: 0.0000

Unidades: Metros

Figura 1. Área de estudio y Puntos del levantamieto topográfico.

\section{Clasificación geomorfológica usando TPI}

El índice de posición topográfica o TPI (Topographic Position Index) permite describir aspectos morfológicos del terreno a través del cálculo y sectorización de las pendientes. El TPI es un método de clasificación de la posición de la pendiente y de tipos de geomorfología.

El valor del TPI viene dado por:

$$
\boldsymbol{T P I _ { i }}=\left(\boldsymbol{h}_{i}-\mu_{i}\right) / \sigma_{i}
$$

En donde el TPI $\boldsymbol{I}_{i}$ se calcula para el i-ésimo pixel del DEM y $\boldsymbol{h}_{i}$ se refiere a la elevación de dicho pixel. $\boldsymbol{\mu}_{i}$ es el valor promedio de todos los pixeles alrededor del i-ésimo pixel dentro de un cierto rango de distancia. Para este estudio se consideró un rango entre 20 m y 50 m, ya que esta escala es consistente con la extensión promedio de los valles y cimas de acuerdo a la experiencia adquirida en campo durante el levantamiento topográfico. Escalas más grandes 
que esta provocarían la pérdida de las unidades geomorfológicas más pequeñas. De la misma manera, $\sigma_{\mathrm{i}}$ es la desviación estandar de los pixeles del DEM dentro de ese mismo rango. Por lo tanto el TPI es una comparación estadística del valor de elevación de los pixeles respecto a los pixeles en las cercanías. El resultado del TPI depende de la escala ya que utiliza valores de sus cercanías, es decir depende de las longitudes medidas y de la posición de una pendiente (Weiss, 2001). Valores positivos representan ubicaciones que son más elevadas que sus alrededores (Cimas). Valores negativos representan ubicaciones que son más bajas que los alrededores (Valles). Valores cercanos a cero pueden ser áreas planas (con pendiente cerca de cero) o áreas de pendiente constante, donde puede usarse un criterio del ángulo de la pendiente para estimar si se trata de uno o de otro caso.

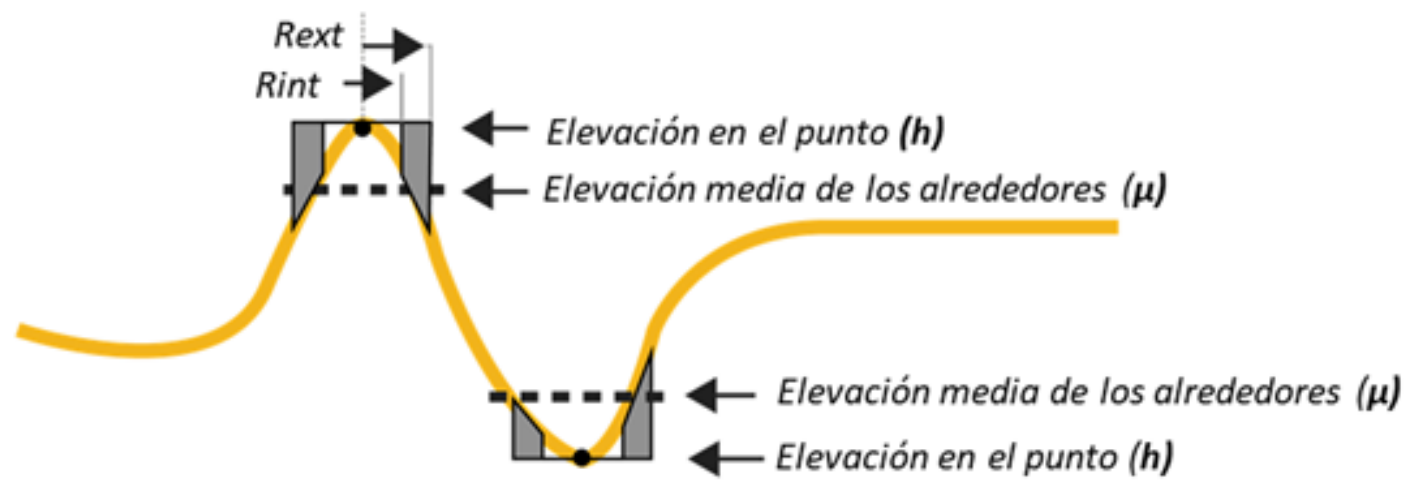

En Pendiente

Elevación media de los alrededores $(\boldsymbol{\mu})$

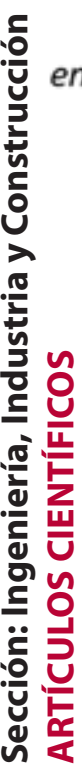

En Planicie

Elevación media de los alrededores $(\boldsymbol{\mu})$

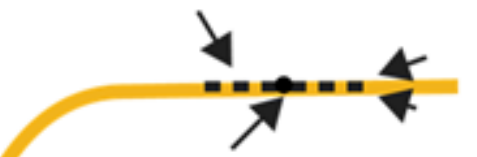

Elevación en el punto (h)

Rext: radio exterior del anillo en las celdas Rint: radio interno del anillo en las celdas

Figura 2. Conceptualización del TPI (modificado de Weiss, 2001)

El TPI genera un mapa de valores continuos. Para el análisis geomorfológico es de mucha utilidad definir umbrales para clasificar formas de terreno. En este trabajo se usaron como umbrales los valores sugeridos por Weiss, 2001, que define seis formas del terreno:

I. Ridges (Cimas): TPI > 1.0 .

II. Upper slope (Pendiente pronunciada): $1.0>\mathrm{TPI}>0.5$ 
III. Middle slope (Pendiene media): 0.5 > TPI > -0.5 (ángulos mayores a 15²)

IV. Flat (Planicie): $0.5>$ TPI > -0.5 (ángulos menores a 15²)

V. Lower slope (Pendiene suave): $-0.5>\mathrm{TPI}>-1.0$

VI. Valley (Valle): TPI<-1.0

\section{RESULTADOS}

\section{Indices de biodividersidad}

Las formaciones vegetales censadas, según el índice de Margalef, indica que el sitio de mayor riqueza es el bosque seco que rodea el manglar y zonas aledañas al rio; el valor más bajo corresponde al manglar, donde la salinidad, inestabilidad del suelo y las inundaciones estacionales, permite que pocas especies proliferen. Según el índice de Simpson, que evalúa la dominancia, señala al manglar como dominante, por la presencia de especies halófilas (toleran la salinidad) que son las que abundan, señalando a las demás formaciones vegetales con una dominancia moderada. El índice de Shannon-Weinner, indica que la playa y bosque seco, tienen una diversidad casi similar, con la disyuntiva que las especies y biotipos son disímiles. Las especies costeras en su mayoría son hierbas y arbustos que crecen en las dunas costeras y suelos salinizados. El bosque seco se compone en su mayoría de árboles que necesitan suelos profundos y un pH neutro; finalmente el índice certifica, que la diversidad vegetal en el manglar es baja.

Tabla 1. Indices de Biodiversidad

\begin{tabular}{|l|l|l|l|}
\hline $\begin{array}{c}\text { Formación } \\
\text { vegetal }\end{array}$ & $\begin{array}{c}\text { Índice de Shannon } \\
\text { (Diversidad) }\end{array}$ & $\begin{array}{c}\text { Índice de Simpson } \\
\text { (Dominancia) }\end{array}$ & $\begin{array}{c}\text { Índice de Margalef } \\
\text { (Riqueza de especies) }\end{array}$ \\
\hline Playa & 2.81 & 0.085 & 3.472 \\
\hline Bosque seco & 2.71 & 0.128 & 5.086 \\
\hline Manglar & 1.58 & 0.2777 & 1.507 \\
\hline
\end{tabular}

\section{Especies vegetales}

\section{Manglar de Ostional}

El sitio muestreado consta de 55 especies censadas, ubicadas en 25 familias botánicas. Dentro de los puntos de muestreo se caracterizaron 3 asociaciones vegetales marcadas las cuales son:

1. Vegetación costera,

2. Manglar, 


\section{3. y bosque secundario seco.}

El manglar se caracteriza por presentar áreas inundables con mezcla de agua dulce y salada. Estos sitios están ocupados por especies halófilas o tolerante a altas concentraciones de sales y al estrés hídrico. Las especies presente en este sitio son Rhizophora mangle L (rojo), Laguncularia racemosa (L.) C.F. Gaertn (blanco), Pelliciera rhizophorae Planch. \& Triana. (piñuela), Hippomane mancinella L., Prosopis juliflora (Sw.) DC., Conocarpus erectus L. (botoncillo), Coccoloba caracasana Meisn., Bromelia pinguin L., Talipariti tiliaceum (Arruda) Fryxell. Estas especies son características de zonas de manglar en el Pacífico de Nicaragua, con la excepción de Pelliciera rhizophorae que no se había reportado en el Pacífico de Nicaragua; pero se conoce que se encuentra distribuido en el zona Caribe de Nicaragua en Bluefileds (Roth 1991). Extrañamente esta planta no ha seguido su distribución y dispersión natural ya que este manglar está confinado por una cresta de arena impidiendo su dispersión hidrocórica marina.

Los índices de diversidad en los manglares son bajos, por ende en esta investigación nos limitamos a mencionar la riqueza de especies en el sitio. Los árboles que se encuentran allí son bastantes maduros con alturas mayores a 12 metros con abundantes árboles reclutas que vienen posicionados de las áreas descubiertas de mangle. 

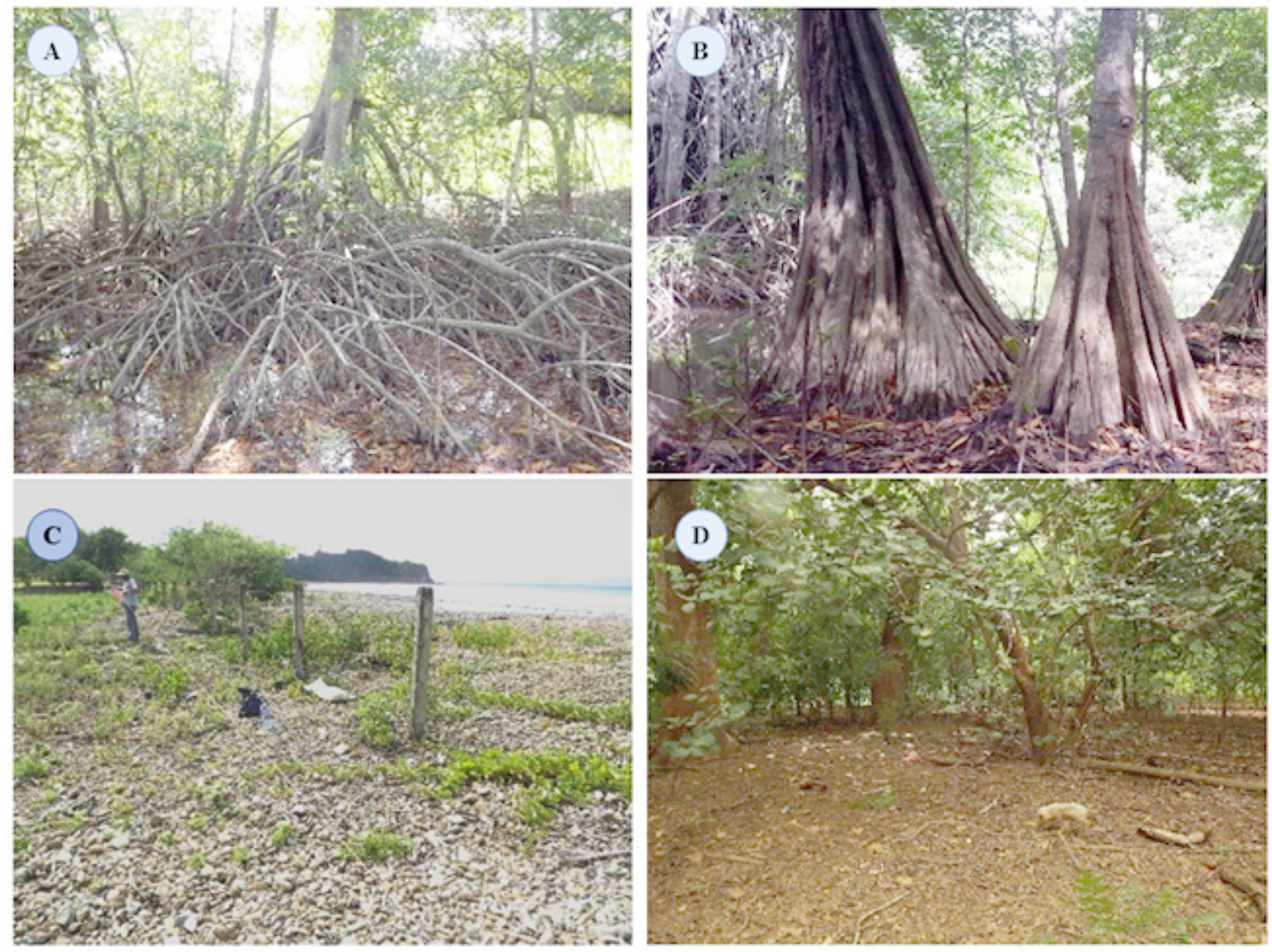

Figura 3. Especies vegetativas en el estuario de Ostional. a) Mangle rojo b) Mangle piñuela c) Vegetación costera c) Bosque seco

\section{Área costera del Ostional}

Se caracteriza por tener especies de arbustal o latizal pioneras, limitadas principalmente a la presencia de arena marina, piedras, residuos calcáreos y carencia de suelo. Las áreas que están expuestas a radiaciones solares altas en su mayoría presentan plantas anuales con un rango de vida corto, estas especies están limitadas a los bancos de arenas. El índice de Shannon $(2,81)$ demuestra que la diversidad en las áreas de marea son medianamente elevados, superiores al manglar y en comparación con el bosque y el índice de Simpson nos indica que existe poca dominancia de las especies que se desarrollan en esa área probablemente por la capacidad de ser una formación que no tiene un equilibrio vegetal al estar muchas especies compitiendo por el espacio.

\section{Bosque seco}

El bosque seco que se presenta a las orillas del río Ostional presenta áreas con árboles con alturas superiores a los 15 metros con una diversidad según Shannon (2.71) menor que 
la de la zona costera con la gran diferencia de que el bosque consta de especímenes de alturas superiores, su diversidad es menor por el patrón de equilibrio de árboles que tienen larga vida y son perennes. En cuanto a especies dominantes, no existe resultados según el índice de Simpson que muestre un patrón de especies dominantes en las poblaciones censadas, manteniéndose la diversidad inversamente proporcional a la dominancia reflejada en las tablas.

\section{Clasificación geomorfológica basada en el TPI}

Como se señaló anteriormente, el índice de posición topográfica es inherentemente dependiente de la escala (Weiss, 2001), y el TPI de un mismo punto podría cambiar según su escala. Entonces, el TPI con un tamaño de análisis pequeño captura las Cimas y Valles locales, mientras que los análisis grandes capturan características a gran escala (Janness, 2006). Dado que el terreno no es muy extenso (aproximadamente $300 \mathrm{~m} \times 600 \mathrm{~m}$ ), es preferente usar una escala pequeña (50 $\mathrm{m}$ de radio).

De acuerdo a esta clasificación, las zonas de planicies rodeadas de Cimas serían las que acumulen más agua dulce (proveniente de precipitación o desbordamiento del río). Las Cimas son zonas de poca acumulación de agua dulce, al estar por encima de las áreas circundantes. Las áreas de pendiente pronunciada son zonas intermedias, donde no se espera mucha acumulación de agua, pero sí se espera escorrentía superficial. 


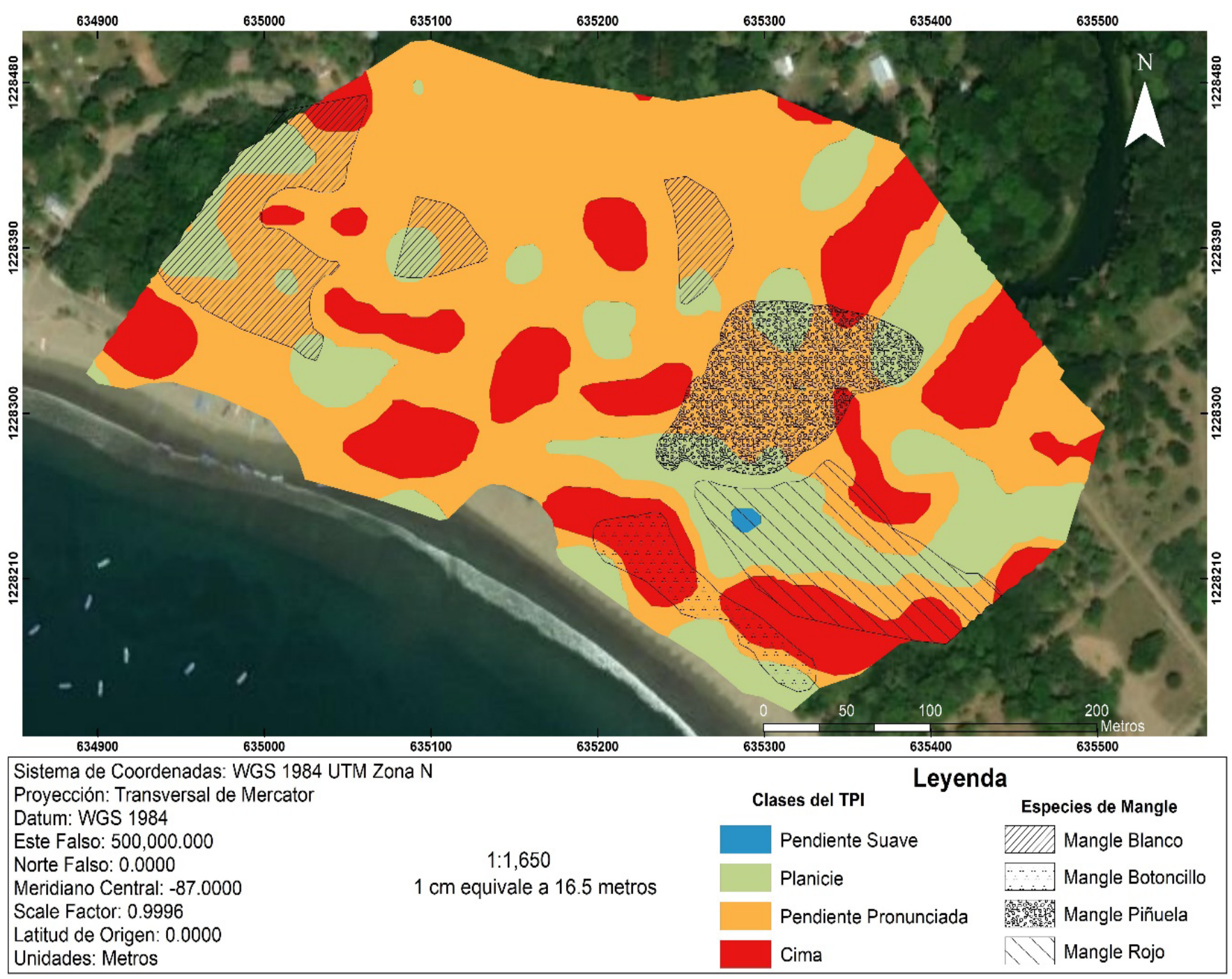

Figura 4. Mapa de clasificación geomorfológica y zonificación de especies de Mangle

\section{DISCUSIÓN}

\section{Inundación y zonificación de especies de mangle}

La contribución de agua dulce al sistema del manglar se da a través de: precipitación local y por inundación desde el estero (deVries 2016; Calderón 2014). Estos aportes de agua dulce se acumulan gracias a el contenido arcilloso de los suelos en zonas del bosque donde hay pequeñas depresiones o barreras formadas por depósitos de sedimentos (deVries 2016). En este caso usamos la clasificación geomorfológica generada por el TPI como un indicador de zonas donde debido a la pendiente, se espera mayor o menor acumulación de agua dulce.

Se definieron zonas representadas por polígonos donde una especie de mangle es mucho más abundante que las otras (Figura 4). Estos polígonos fueron superpuestos sobre las tipologías 
de formas del terreno definidas con el TPI para analizar patrones. Fuera de los polígonos no pudo establecerse la predominancia de una sola especie, lo que limita nuestro análisis.

Los parches de mangle blanco se encuentran en las areas de Pendiente pronunciada y Planicie (upper slope y flat). El mangle piñuela está en una zona de Pendiente pronunciada, pero rodeado de Cimas y planicies. El mangle rojo está concentrado en una zona de Planicie, rodeada de Cimas. Finalmente el botoncillo se halla en zona de Cimas.

Según Jimenez (1998), en los manglares del Pacífico centroamericano con climas secos estacionales el gradiente de salinidad del suelo aumenta al alejarse de los esteros y canales, ya que la frecuencia de inundación disminuye y hay mayor concentración de sales, lo que se relaciona con un menor desarrollo del tamaño de los árboles. En el caso de Ostional, la barra de arena cierra el sistema al mar y las inundaciones se dan ya sea por el llenado del estero o por exceso de precipitación. El mangle rojo se desarrolla en las zonas más inundables (cerca del estero y la zona de Planicies (flat) rodeada de Cimas (ridges). En este caso, cerca del estero donde tiene un suministro constante de agua. En cambio, el Mangle Blanco se concentra en zonas menos inundables y el Botoncillo crece sobre Cimas (ridges), fuera de la zona de inundación. Esto se corresponde con patrones de zonificación ya conocidos (Figura 5).

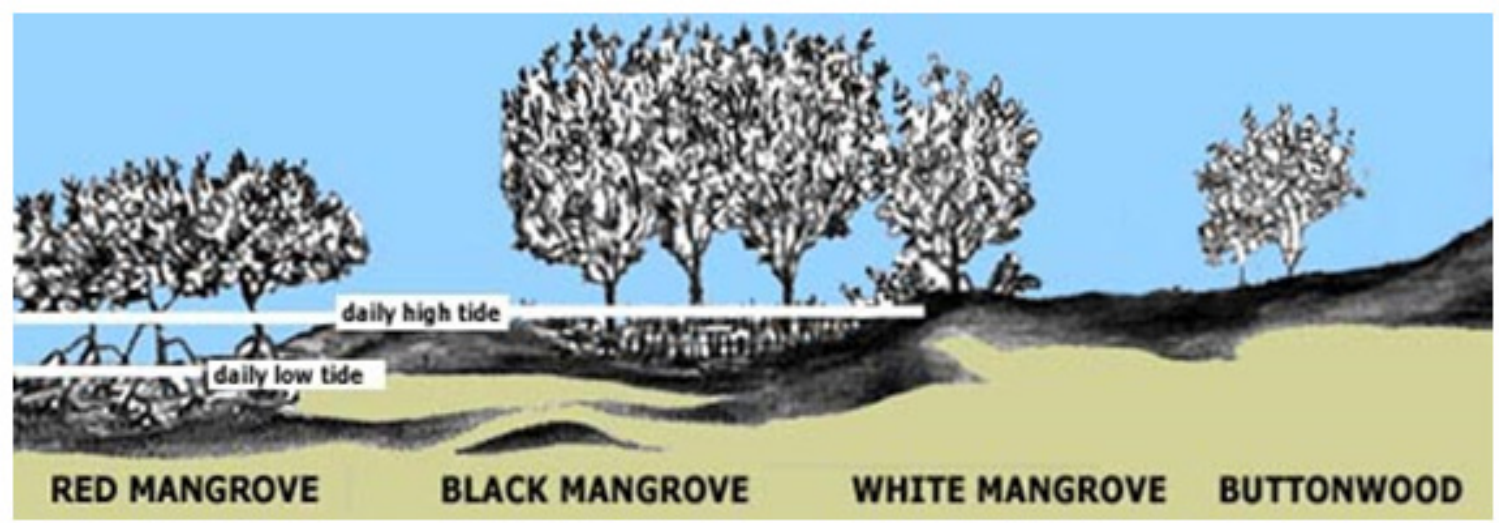

Figura 5. Zonificación de especies de mangle y zonas inundables por la marea (Red mangrove= Mangle rojo, Black Mangrove=mangle negro, White Mangrove=Mangle blanco, Buttonwood=botoncillo) (https://www. floridamuseum.ufl.edu/southflorida/habitats/mangroves/zonation/)

La presencia del mangle piñuela está asociada al suministro de agua fresca. En ambientes no perturbados es un constituyente menor pero tiende a propagarse si los otros árboles han sido removidos y el agua dulce esta disponible. Este es el caso en Ostional. Crece mejor en suelos húmedos, inundados lentamente por la marea alta (IUCN 2008). Esta especie está dentro de la lista roja de especies en peligro de IUCN y no había sido reportado para el Pacífico de Nicaragua. 


\section{CONCLUSIONES}

El bosque del estero de Ostional está compuesto por una zona de manglar que incluye: Mangle Blanco, Rojo, Piñuela y Botoncillo. Además se identificó vegetación costera y de bosque tropical seco.

El TPI permitió una adecuada clasificación de formas de terreno en una zona con poca variación de elevaciones ( $3 \mathrm{~m})$. La zonificación de especies de mangle en esta zona esta asociada a las areas inundables (Planicies rodeadas de Cimas): mangle rojo. El Mangle Blanco se ubica en zonas de Pendiente pronunciada donde hay menos inundación y el botoncillo en zonas de Cimas donde no ocurre inundación.

El mangle piñuela ha podido propagarse debido al suministro de agua dulce del estero y a la remoción de otras especies por actividad antrópica. Esta especie está dentro de la lista roja de especies en peligro de IUCN y no había sido reportado para el Pacífico de Nicaragua.

El uso de TPI como herramienta de clasificación de formas de terreno es una herramienta útil aun en el caso de pequeñas variaciones en las elevaciones y pequeñas escalas.

\section{AGRADECIMIENTOS}

El financiamiento para este trabajo fue proporcionado por los Fondos para Investigación (FPI) de la UNAN-Managua. El levantamiento topográfico fue hecho por la Facultad de Ciencias e Ingeniería con la ayuda del Ing. Roberto Aguirre.

\section{BIBLIOGRAFÍA}

Araujo, A; Bascopé, F; Cardona, V; De la Quintana, D; Fuentes, A; Jørgensen, P; Maldonado, C; Miranda, T; Paniagua, N; \& Seidel, R. 2005. Composición florística y estructura del bosque amazónico preandino en el sector del Arroyo Negro, Parque Nacional Madidi, Bolivia; Ecología en Bolivia, Vol. 40(3): 281-303.

Calderon, H., Weeda, R., \& Uhlenbrook, S. (2014). Hydrological and geomorphological controls on the water balance components of a mangrove forest during the dry season in the Pacific Coast of Nicaragua. Wetlands, 34(4), 685-697.
De Vries, S. (2016). Blind estuaries during drought: The influences of a sandbar on mangrove trees. Tesis de Maestría. TU Delft.

Ellison, A., Farnsworth, E. \& Moore, G. 2010. Pelliciera rhizophorae. The IUCN Red List of Threatened Species 2010: e.T178833A7621318. http://dx.doi. org/10.2305/IUCN.UK.20102.RLTS. T178833A7621318.en

Feller, I \& Sitnik, M. 1996. Mangrove ecology: A manual for a field course, A fields manual focused on the biocomplexity on mangrove ecosystem. Smithsonian institution. 1-45. 
Foster, B. R., N. C. Hernández, E., E. K. Kakudidi y R. J. Burnham. 1995. Un método de transectos variables para la evaluación rápida de comunidades de plantas en los trópicos. Manuscrito no publicado. Chicago: Environmental and Conservation Programs, Field Museum of Natural History; and Washington, D. C.: Conservation Biology, Conservation International.

He, G., Engel, V., Leonard, L., Croft, A., Childers, D., Laas, M., Deng, Y. and SoloGabriele, H. 2010. Factors controlling surface water flow in a low gradient subtropical wetland. Wetlands, 30(2), 275-286.

Jenness, J. 2006. Topographic position index (TPI) v. 1.2. Jenness Enterprises.

Jiménez, J. A., 1992. Mangrove forests of the Pacific coast of Central America. Coastal plant communities of Latin America, 259-267.

Jiménez, J. A., 1994. Los manglares del Pacífico centroamericano (No. 333.91809728 J61). Editorial Fundación UNA.

Jiménez, J. A., Yañez-Arancibia, A. y Lara-Rodriguez, A., 1999. Ambiente, distribucion y caracteristicas estructurales de los manglares del Pacifico de Centro America: contrastes climaticos. En: Yañez-Arancibia, A y Lara-Rodriguez, A. eds. Ecosistemas de manglar en America Tropical. 1era ed. Mexico: Instituto de Ecologia A.C. Centro SEP-CONACYT, 51-70.
Kathiresan, K. 2008. Importance of mangrove ecosystem. Centre of advanced study in marine biology. Annamalai University.1-34.

Lugo, A.E. y Snedaker, S.C. 1974. The ecology of mangroves. Annual Review of Ecology and Sustematics, 5, 39-64.

Matteucc, Sy Colma A. 1982. Metodología para el estudio de la vegetación. Departamento de asuntos científicos y tecnológicos de la secretaria general de la organización de los estados americanos. 25. Estado de Falcón, Venezuela.

Mostacedo B \& Fredericksen, T. 2000. Manual de métodos básicos de muestreo y análisis en ecología vegetal Editora El País, Bolivia.

Pineda M, M. D. (2015). Evaluación de metodos de interpolación, para generación de modelos digitales de elevacion, en areas planas. Researchgate.

Reyes, M y Tovilla, C.2002. Restauración de áreas alteradas de manglar con Rhizophora mangle en la Costa de Chiapas. Madera y Bosques Número especial, 103-114.

Stevens, W.D., Ulloa, C., Pool, A. y Montiel, O.M. (Eds.). 2001. Flora de Nicaragua. Vol. 85, tomos I, II y III. Missouri Botanical Garden Press, St. Louis Missouri.

Tomlinson, P. 1986. The botany of mangroves. Cambridge University Press, Cambridge, United Kingdom.413.

Vílchez, J. (2000). Evaluación de la exactitud de modelos de elevación digital (MED) de malla regular generados a partir de curvas 
de nivel. Revista Geográfica Venezolana, 41(2), 239-256.

Villatoro, M. H. (2008). Comparación de los interpoladores IDW y Kriging en la variación espacial de $\mathrm{pH}, \mathrm{Ca}, \mathrm{CICE}$ y $\mathrm{P}$ del suelo. Agronomía Costarricense, 32(1), 95-105.
Weiss, A. (2001, July). Topographic position and landforms analysis. In Poster presentation, ESRI user conference, San Diego, CA (Vol. 200).

\section{ANEXO}

Tabla 1. Especies presente en Ostional

\begin{tabular}{|c|c|}
\hline Especies & Familia botánica \\
\hline Annona reticulata L. & Annonaceae \\
\hline Cascabela ovata (Cav.) Lippold & Apocynaceae \\
\hline Rauvolfia tetraphylla L. & Apocynaceae \\
\hline Stemmadenia pubescens Benth. & Apocynaceae \\
\hline Crescentia alata Kunth & Bignoniaceae \\
\hline Crescentia cujete L. & Bignoniaceae \\
\hline Tabebuia rosea (Bertol.) DC. & Bignoniaceae \\
\hline Cordia dentata Poir & Boraginaceae \\
\hline Varronia inermis (Mill.) Borhidi & Boraginaceae \\
\hline Bromelia pinguin L. & Bromeliaceae \\
\hline Cleome spinosa Jacq. & Capparaceae \\
\hline Conocarpus erectus L. & Combretaceae \\
\hline Laguncularia racemosa (L.) C.F. Gaertn. & Combretaceae \\
\hline Commelina erecta L. & Commelinaceae \\
\hline Ipomoea pes-caprae (L.) R. Br. & Convolvulaceae \\
\hline Euphorbia hirta L. & Euphorbiaceae \\
\hline Hippomane mancinella L. & Euphorbiaceae \\
\hline Hura crepitans L. & Euphorbiaceae \\
\hline Jatropha gossypiifolia L. & Euphorbiaceae \\
\hline
\end{tabular}




\begin{tabular}{|c|c|}
\hline Especies & Familia botánica \\
\hline Sapium glandulosum (L.) Morong & Euphorbiaceae \\
\hline Canavalia rosea (Sw.) DC. & Fabaceae \\
\hline Crotalaria retusa L. & Fabaceae \\
\hline Enterolobium cyclocarpum (Jacq.) Griseb & Fabaceae \\
\hline Gliricidia sepium (Jacq.) Kunth & Fabaceae \\
\hline Haematoxylum brasiletto H. Karst & Fabaceae \\
\hline Mimosa pudica L. & Fabaceae \\
\hline $\begin{array}{l}\text { Pithecellobium lanceolatum (Humb. \& Bonpl. ex } \\
\text { Willd.) Benth. }\end{array}$ & Fabaceae \\
\hline Prosopis juliflora (Sw.) DC. & Fabaceae \\
\hline Samanea saman (Jacq.) Merr. & Fabaceae \\
\hline Senna atomaria L. & Fabaceae \\
\hline Senna pallida (Vahl) H.S. Irwin \& Barneby & Fabaceae \\
\hline Tamarindus indica L & Fabaceae \\
\hline Vachellia collinsii (Saff.) Seigler \& Ebinger & Fabaceae \\
\hline Vachellia farnesiana (L.) Wight \& Arn. & Fabaceae \\
\hline Astraea lobata (L.) Klotzsch & Malvaceae \\
\hline Guazuma ulmifolia Lam. & Malvaceae \\
\hline Sida sp. & Malvaceae \\
\hline Talipariti tiliaceum (Arruda) Fryxell & Malvaceae \\
\hline Waltheria indica L. & Malvaceae \\
\hline Azadirachta indica A. & Meliaceae \\
\hline Trichilia martiana C. DC. & Meliaceae \\
\hline Castilla elastica Sessé & Moraceae \\
\hline Pisonia macranthocarpa (Donn. Sm.) Donn. Sm. & Nyctaginaceae \\
\hline Passiflora foetida L. & Passifloraceae \\
\hline Turnera scabra Millsp & Passifloraceae \\
\hline
\end{tabular}




\begin{tabular}{|l|l|}
\hline \multicolumn{1}{|c|}{ Especies } & \multicolumn{1}{|c|}{ Familia botánica } \\
\hline Petiveria alliacea L. & Phytolaccaceae \\
\hline Piper sp. & Piperaceae \\
\hline Coccoloba caracasana Meisn. & Polygonaceae \\
\hline Coccoloba floribunda (Benth.) Lindau & Polygonaceae \\
\hline Rhizophora mangle L. & Rhizophoraceae \\
\hline Thouinidium decandrum (Bonpl.) Radlk. & Sapindaceae \\
\hline Simarouba amara Aubl. & Simaroubaceae \\
\hline Pelliciera rhizophorae Planch. \& Triana & Tetrameristaceae \\
\hline Cissus verticillata (L.) Nicolson \& C.E. Jarvis & Vitaceae \\
\hline
\end{tabular}

\section{Manglar}

\begin{tabular}{|l|l|}
\hline \multicolumn{1}{|c|}{ Especie } & \multicolumn{1}{c|}{ Familia } \\
\hline Rhizophora mangle L. & Rhizophoraceae \\
\hline Laguncularia racemosa (L.) C.F. Gaertn. & Combretaceae \\
\hline Pelliciera rhizophorae Planch. \& Triana & Tetrameristaceae \\
\hline Hippomane mancinella L. & Euphorbiaceae \\
\hline Prosopis juliflora (Sw.) DC. & Fabaceae \\
\hline Conocarpus erectus L. & Combretaceae \\
\hline Coccoloba caracasana Meisn. & Polygonaceae \\
\hline Bromelia pinguin L. & Bromeliaceae \\
\hline Talipariti tiliaceum (Arruda) Fryxell & Malvaceae \\
\hline
\end{tabular}

Tabla 2. Con la aplicación de los índices de diversidad presente en el área costera de las playas de Ostional

\begin{tabular}{|l|l|l|l|l|}
\hline \multicolumn{1}{|c|}{ Especie } & ni & \multicolumn{1}{c|}{ Pi } & Ln (Pi) & \multicolumn{1}{c|}{$\mathbf{H}^{\prime}$} \\
\hline Astraea lobata (L.) Klotzsch & 2 & 0,008403361 & $-4,779123493$ & $-0,040160702$ \\
\hline Azadirachta indica A. & 19 & 0,079831933 & $-2,527831695$ & $-0,20180169$ \\
\hline Crescentia cujete L. & 3 & 0,012605042 & $-4,373658385$ & $-0,055130148$ \\
\hline Canavalia rosea (Sw.) DC. & 6 & 0,025210084 & $-3,680511204$ & $-0,092785997$ \\
\hline
\end{tabular}




\begin{tabular}{|c|c|c|c|c|}
\hline Especie & ni & $\mathbf{P i}$ & $\operatorname{Ln}(\mathbf{P i})$ & $\mathbf{H}^{\prime}$ \\
\hline $\begin{array}{l}\text { Cascabela ovata (Cav.) Li- } \\
\text { ppold }\end{array}$ & 2 & 0,008403361 & $-4,779123493$ & $-0,040160702$ \\
\hline $\begin{array}{l}\text { Cissus verticillata (L.) Nicol- } \\
\text { son \& C.E. Jarvis }\end{array}$ & 1 & 0,004201681 & $-5,472270674$ & $-0,022992734$ \\
\hline Cleome spinosa Jacq. & 4 & 0,016806723 & $-4,085976313$ & $-0,068671871$ \\
\hline Commelina erecta L. & 4 & 0,016806723 & $-4,085976313$ & $-0,068671871$ \\
\hline Crotalaria retusa L. & 2 & 0,008403361 & $-4,779123493$ & $-0,040160702$ \\
\hline Euphorbia hirta L. & 16 & 0,067226891 & $-2,699681951$ & $-0,181491224$ \\
\hline Guazuma ulmifolia Lam. & 5 & 0,021008403 & $-3,862832761$ & $-0,081151949$ \\
\hline $\begin{array}{l}\text { Haematoxylum brasiletto } \mathrm{H} \text {. } \\
\text { Karst }\end{array}$ & 10 & 0,042016807 & $-3,169685581$ & $-0,133180066$ \\
\hline Hippomane mancinella L. & 1 & 0,004201681 & $-5,472270674$ & $-0,022992734$ \\
\hline Ipomoea pes-caprae (L.) R. Br. & 10 & 0,042016807 & $-3,169685581$ & $-0,133180066$ \\
\hline Jatropha gossypiifolia L. & 26 & 0,109243697 & $-2,214174136$ & $-0,241884569$ \\
\hline Mimosa pudica L. & 3 & 0,012605042 & $-4,373658385$ & $-0,055130148$ \\
\hline Passiflora foetida L. & 2 & 0,008403361 & $-4,779123493$ & $-0,040160702$ \\
\hline Petiveria alliacea L. & 2 & 0,008403361 & $-4,779123493$ & $-0,040160702$ \\
\hline Rauvolfia tetraphylla L. & 3 & 0,012605042 & $-4,373658385$ & $-0,055130148$ \\
\hline $\begin{array}{l}\text { Senna pallida (Vahl) H.S. } \\
\text { Irwin \& Barneby }\end{array}$ & 23 & 0,096638655 & $-2,336776458$ & $-0,225822935$ \\
\hline Sida sp. & 9 & 0,037815126 & $-3,275046096$ & $-0,123846281$ \\
\hline Simarouba amara Aubl. & 2 & 0,008403361 & $-4,779123493$ & $-0,040160702$ \\
\hline $\begin{array}{l}\text { Stemmadenia pubescens } \\
\text { Benth. }\end{array}$ & 3 & 0,012605042 & $-4,373658385$ & $-0,055130148$ \\
\hline Tamarindus indica L & 4 & 0,016806723 & $-4,085976313$ & $-0,068671871$ \\
\hline Turnera scabra Millsp & 22 & 0,092436975 & $-2,38122822$ & $-0,220113533$ \\
\hline $\begin{array}{l}\text { Vachellia collinsii (Saff.) Sei- } \\
\text { gler \& Ebinger }\end{array}$ & 45 & 0,18907563 & $-1,665608184$ & $-0,314925917$ \\
\hline
\end{tabular}




\begin{tabular}{|l|l|l|l|l|}
\hline \multicolumn{1}{|c|}{ Especie } & ni & \multicolumn{1}{c|}{ Pi } & Ln (Pi) & H' $^{\prime}$ \\
\hline $\begin{array}{l}\text { Varronia inermis (Mill.) } \\
\text { Borhidi }\end{array}$ & 5 & 0,021008403 & $-3,862832761$ & $-0,081151949$ \\
\hline Waltheria indica L. & 4 & 0,016806723 & $-4,085976313$ & $-0,068671871$ \\
\hline & 238 & & & $\mathbf{- 2 , 8 1 3 4 9 3 9 2 8}$ \\
\hline
\end{tabular}

Tabla 3. Explica índice de Shannon para la diversidad en el bosque seco presente en cercanías del rio de Ostional

\begin{tabular}{|l|l|l|l|l|}
\hline \multicolumn{1}{|c|}{ Especie } & ni & \multicolumn{1}{c|}{ pi } & \multicolumn{1}{c|}{ Ln(pi) } & H' $^{\prime}$ \\
\hline Annona reticulata L. & 2 & 0,0645161 & $-2,74084$ & $-0,176828$ \\
\hline Azadirachta indica A. & 31 & 0,2695652 & $-1,310945$ & $-0,353385$ \\
\hline Castilla elastica Sessé & 1 & 0,0086957 & $-4,744932$ & $-0,04126$ \\
\hline $\begin{array}{l}\text { Cissus verticillata (L.) Nicolson \& C.E. } \\
\text { Jarvis }\end{array}$ & 1 & 0,0086957 & $-4,744932$ & $-0,04126$ \\
\hline Coccoloba caracasana Meisn. & 2 & 0,0173913 & $-4,051785$ & $-0,070466$ \\
\hline Coccoloba floribunda (Benth.) Lindau & 1 & 0,0086957 & $-4,744932$ & $-0,04126$ \\
\hline Cordia dentata Poir & 1 & 0,0086957 & $-4,744932$ & $-0,04126$ \\
\hline Crescentia alata Kunth & 1 & 0,0086957 & $-4,744932$ & $-0,04126$ \\
\hline Crescentia cujete L. & 1 & 0,0086957 & $-4,744932$ & $-0,04126$ \\
\hline $\begin{array}{l}\text { Enterolobium cyclocarpum (Jacq.) } \\
\text { Griseb }\end{array}$ & 1 & 0,0086957 & $-4,744932$ & $-0,04126$ \\
\hline Gliricidia sepium (Jacq.) Kunth & 2 & 0,0173913 & $-4,051785$ & $-0,070466$ \\
\hline Guazuma ulmifolia Lam. & 1 & 0,0086957 & $-4,744932$ & $-0,04126$ \\
\hline Hura crepitans L. & 3 & 0,026087 & $-3,64632$ & $-0,095121$ \\
\hline Piper sp. & 2 & 0,0173913 & $-4,051785$ & $-0,070466$ \\
\hline $\begin{array}{l}\text { Pisonia macranthocarpa (Donn. Sm.) } \\
\text { Donn. Sm. }\end{array}$ & 1 & 0,0086957 & $-4,744932$ & $-0,04126$ \\
\hline $\begin{array}{l}\text { Pithecellobium lanceolatum (Humb. \& } \\
\text { Bonpl. ex Willd.) Benth. }\end{array}$ & 4 & 0,0347826 & $-3,358638$ & $-0,116822$ \\
\hline Prosopis juliflora (Sw.) DC. & 5 & 0,0434783 & $-3,135494$ & $-0,136326$ \\
\hline
\end{tabular}




\begin{tabular}{|l|l|l|l|l|}
\hline \multicolumn{1}{|c|}{ Especie } & ni & pi & Ln(pi) & \multicolumn{1}{c|}{$\mathbf{H}^{\prime}$} \\
\hline Samanea saman (Jacq.) Merr. & 1 & 0,0086957 & $-4,744932$ & $-0,04126$ \\
\hline Sapium glandulosum (L.) Morong & 1 & 0,0086957 & $-4,744932$ & $-0,04126$ \\
\hline Senna atomaria L. & 1 & 0,0086957 & $-4,744932$ & $-0,04126$ \\
\hline Simarouba amara Aubl. & 2 & 0,0173913 & $-4,051785$ & $-0,070466$ \\
\hline Stemmadenia pubescens Benth. & 4 & 0,0347826 & $-3,358638$ & $-0,116822$ \\
\hline Tabebuia rosea (Bertol.) DC. & 1 & 0,0086957 & $-4,744932$ & $-0,04126$ \\
\hline $\begin{array}{l}\text { Thouinidium decandrum (Bonpl.) } \\
\text { Radlk. }\end{array}$ & 12 & 0,1043478 & $-2,260025$ & $-0,235829$ \\
\hline Trichilia martiana C. DC. & 11 & 0,0956522 & $-2,347037$ & $-0,224499$ \\
\hline $\begin{array}{l}\text { Vachellia collinsii (Saff.) Seigler \& } \\
\text { Ebinger }\end{array}$ & 14 & 0,1217391 & $-2,105875$ & $-0,256367$ \\
\hline Vachellia farnesiana (L.) Wight \& Arn. & 8 & 0,0695652 & $-2,665491$ & $-0,185425$ \\
\hline & 115 & & & $-\mathbf{2 , 7 1 5 6 7 3}$ \\
\hline
\end{tabular}

Índice de Simpson donde demuestra y apoya la diversidad existente en el área donde no existe plantas dominante en los censos realizados en la zona costera.

\begin{tabular}{|l|l|l|l|}
\hline \multicolumn{1}{|c|}{ Especie } & ni & \multicolumn{1}{c|}{ Pi } & \multicolumn{1}{c|}{ Pi ^2 } \\
\hline Astraea lobata (L.) Klotzsch & 2 & 0,008403361 & $7,06165 \mathrm{E}-05$ \\
\hline Azadirachta indica A. & 19 & 0,079831933 & 0,006373137 \\
\hline Crescentia cujete L. & 3 & 0,012605042 & 0,000158887 \\
\hline Canavalia rosea (Sw.) DC. & 6 & 0,025210084 & 0,000635548 \\
\hline Cascabela ovata (Cav.) Lippold & 2 & 0,008403361 & $7,06165 \mathrm{E}-05$ \\
\hline $\begin{array}{l}\text { Cissus verticillata (L.) Nicolson \& C.E. } \\
\text { Jarvis }\end{array}$ & 1 & 0,004201681 & $1,76541 \mathrm{E}-05$ \\
\hline Cleome spinosa Jacq. & 4 & 0,016806723 & 0,000282466 \\
\hline Commelina erecta L. & 4 & 0,016806723 & 0,000282466 \\
\hline Crotalaria retusa L. & 2 & 0,008403361 & $7,06165 \mathrm{E}-05$ \\
\hline Euphorbia hirta L. & 16 & 0,067226891 & 0,004519455 \\
\hline
\end{tabular}




\begin{tabular}{|c|c|c|c|}
\hline Especie & ni & $\mathbf{P i}$ & $\mathbf{P i} \wedge 2$ \\
\hline Guazuma ulmifolia Lam. & 5 & 0,021008403 & 0,000441353 \\
\hline Haematoxylum brasiletto H. Karst & 10 & 0,042016807 & 0,001765412 \\
\hline Hippomane mancinella L. & 1 & 0,004201681 & 1,76541E-05 \\
\hline Ipomoea pes-caprae (L.) R. Br. & 10 & 0,042016807 & 0,001765412 \\
\hline Jatropha gossypiifolia L. & 26 & 0,109243697 & 0,011934185 \\
\hline Mimosa pudica L. & 3 & 0,012605042 & 0,000158887 \\
\hline Passiflora foetida L. & 2 & 0,008403361 & $7,06165 \mathrm{E}-05$ \\
\hline Petiveria alliacea L. & 2 & 0,008403361 & $7,06165 \mathrm{E}-05$ \\
\hline Rauvolfia tetraphylla L. & 3 & 0,012605042 & 0,000158887 \\
\hline Senna pallida (Vahl) H.S. Irwin \& Barneby & 23 & 0,096638655 & 0,00933903 \\
\hline Sida sp. & 9 & 0,037815126 & 0,001429984 \\
\hline Simarouba amara Aubl. & 2 & 0,008403361 & $7,06165 \mathrm{E}-05$ \\
\hline Stemmadenia pubescens Benth. & 3 & 0,012605042 & 0,000158887 \\
\hline Tamarindus indica $\mathrm{L}$ & 4 & 0,016806723 & 0,000282466 \\
\hline Turnera scabra Millsp & 22 & 0,092436975 & 0,008544594 \\
\hline Vachellia collinsii (Saff.) Seigler \& Ebinger & 45 & 0,18907563 & 0,035749594 \\
\hline Varronia inermis (Mill.) Borhidi & 5 & 0,021008403 & 0,000441353 \\
\hline \multirow[t]{3}{*}{ Waltheria indica L. } & 4 & 0,016806723 & 0,000282466 \\
\hline & 238 & D & 0,085163477 \\
\hline & & 1-D & 0,914836523 \\
\hline
\end{tabular}

El índice de Simpson en el bosque seco donde demuestra de que esta en condiciones de diversidad equilibradas o en búsqueda del equilibrio donde las especies pioneras van desapareciendo para permanecer las perennes.

\begin{tabular}{|l|l|l|l|}
\hline \multicolumn{1}{|c|}{ Especie } & \multicolumn{1}{c|}{ ni } & \multicolumn{1}{c|}{ pi } & \multicolumn{1}{c|}{ pi ^2 } \\
\hline Annona reticulata L. & 2 & 0,064516129 & 0,0041623 \\
\hline Azadirachta indica A. & 31 & 0,269565217 & 0,0726654 \\
\hline Castilla elastica Sessé & 1 & 0,008695652 & $7,561 \mathrm{E}-05$ \\
\hline
\end{tabular}




\begin{tabular}{|l|l|l|l|}
\hline \multicolumn{1}{|c|}{ Especie } & ni & \multicolumn{1}{c|}{ pi } & Pi ^2 \\
\hline Cissus verticillata (L.) Nicolson \& C.E. Jarvis & 1 & 0,008695652 & $7,561 \mathrm{E}-05$ \\
\hline Coccoloba caracasana Meisn. & 2 & 0,017391304 & 0,0003025 \\
\hline Coccoloba floribunda (Benth.) Lindau & 1 & 0,008695652 & $7,561 \mathrm{E}-05$ \\
\hline Cordia dentata Poir & 1 & 0,008695652 & $7,561 \mathrm{E}-05$ \\
\hline Crescentia alata Kunth & 1 & 0,008695652 & $7,561 \mathrm{E}-05$ \\
\hline Crescentia cujete L. & 1 & 0,008695652 & $7,561 \mathrm{E}-05$ \\
\hline Enterolobium cyclocarpum (Jacq.) Griseb & 1 & 0,008695652 & $7,561 \mathrm{E}-05$ \\
\hline Gliricidia sepium (Jacq.) Kunth & 2 & 0,017391304 & 0,0003025 \\
\hline Guazuma ulmifolia Lam. & 1 & 0,008695652 & $7,561 \mathrm{E}-05$ \\
\hline Hura crepitans L. & 3 & 0,026086957 & 0,0006805 \\
\hline Piper sp. & 2 & 0,017391304 & 0,0003025 \\
\hline Pisonia macranthocarpa (Donn. Sm.) Donn. Sm. & 1 & 0,008695652 & $7,561 \mathrm{E}-05$ \\
\hline Pithecellobium lanceolatum (Humb. \& ) & 4 & 0,034782609 & 0,0012098 \\
\hline Prosopis juliflora (Sw.) DC. & 5 & 0,043478261 & 0,0018904 \\
\hline Samanea saman (Jacq.) Merr. & 1 & 0,008695652 & $7,561 \mathrm{E}-05$ \\
\hline Sapium glandulosum (L.) Morong & 1 & 0,008695652 & $7,561 \mathrm{E}-05$ \\
\hline Senna atomaria L. & 1 & 0,008695652 & $7,561 \mathrm{E}-05$ \\
\hline Simarouba amara Aubl. & 2 & 0,017391304 & 0,0003025 \\
\hline Stemmadenia pubescens Benth. & 4 & 0,034782609 & 0,0012098 \\
\hline Tabebuia rosea (Bertol.) DC. & 12 & 0,008695652 & $7,561 \mathrm{E}-05$ \\
\hline Thouinidium decandrum (Bonpl.) Radlk. & 11 & 0,095652174 & 0,0091493 \\
\hline Trichilia martiana C. DC. & 14 & 0,12173913 & 0,0148204 \\
\hline Vachellia collinsii (Saff.) Seigler \& Ebinger & $\mathbf{1 1 5}$ & 0,069565217 & 0,0048393 \\
\hline Vachellia farnesiana (L.) Wight \& Arn. & $\mathbf{1}-\mathbf{D}$ & $\mathbf{0 , 1 2 3 7 0 8 6}$ \\
\hline & $\mathbf{0 , 8 7 6 2 9 1 4}$ \\
\hline
\end{tabular}

